\title{
Occurrence of leaf spot disease on date palm caused by Neopestalotiopsis clavispora in Iran
}

\author{
Esmaeil Basavand ${ }^{1} \cdot$ Ali Pakdin-Parizi ${ }^{2} \cdot$ Hossein-Ali Mirhosseini ${ }^{3} \cdot$ Mojtaba Dehghan-Niri $^{4}$
}

Received: 28 July 2019 / Accepted: 27 February 2020 / Published online: 12 March 2020

(C) Società Italiana di Patologia Vegetale (S.I.Pa.V.) 2020

Keywords Date palm $\cdot$ Leaf spot $\cdot$ Neopestalotiopsis clavispora $\cdot$ ITS region

In May 2016, symptoms of circular leaf spots with concentric rings, later developing into irregular lesions were observed on the leaves of date palm (Phoenix dactylifera L.) in Sari County, Iran. Fungal isolations were done based on conventional methods (Mirhosseini et al. 2017). Woolly-cottony white colonies were grown on PDA. After 10 days, black acervuli distributed in concentric circles appeared throughout the entire plate. Conidia were fusiform, straight, and had five cells. Basal and apical cells were hyaline while third and fourth cells were dark brown. All conidia had one basal appendage (average $8.7 \mu \mathrm{m}$ ) and two to four (commonly four) apical appendages (average $23.7 \mu \mathrm{m})$. Morphological identification, corresponding with Neopestalotiopsis clavispora, was confirmed by sequencing of the ITS region amplified by ITS5 and ITS4 primers and comparison with sequences in GenBank. BLAST algorithm showed 100\% homology with $N$. clavispora (Accession No. MH685414.1). Pathogenicity was confirmed by placing six mycelial plugs from the three representative actively growing fungi on the seedling. Two plants, each containing three leaves, were used for this test. Plants were kept in a glasshouse at about $25^{\circ} \mathrm{C}$ with a 14 -h photoperiod. All inoculated leaves developed dark lesions after 8 days of incubation, whereas control leaves remained asymptomatic. There are some reports of $N$. clavispora causing disease on strawberry worldwide

Esmaeil Basavand

e.basavand@vru.ac.ir

1 Department of Plant Pathology, Vali-e-Asr University of Rafsanjan, Rafsanjan, Iran

2 Genetics and Agricultural Biotechnology Institute of Tabarestan, Sari Agricultural Sciences and Natural Resources University, Sari, Iran

3 Department of Plant Pathology, Gorgan University of Agricultural Sciences and Natural Resources, Gorgan, Iran

4 Department of Plant Pathology, Ferdowsi University of Mashhad, Mashhad, Iran
(Chamorro et al. 2016; Mahapatra et al. 2018) and Macadamia in Brazil (Santos et al. 2019), but this is the first report of leaf spot caused by $N$. clavispora on date palm in Iran.

Acknowledgments This research was supported by Vice Chancellor of Research and Technology at the Vali-E-Asr University of Rafsnjan, Iran.

\section{References}

Chamorro M, Aguado A, De los Santos B (2016) First report of root and crown rot caused by Pestalotiopsis clavispora (Neopestalotiopsis clavispora) on strawberry in Spain. Plant Dis 100(7):1495

Mahapatra S, Banerjee J, Kumar K, Pramanik S, Pramanik K, Islam S, Das S (2018) Leaf spot and fruit rot of strawberry caused by Neopestalotiopsis clavispora in Indo-Gangetic plains of India. Indian Phytopathol 1-5

Mirhosseini H, Babaeizad V, Hashemi L, Basavand E (2017) Identification and investigation of genotypic and phenotypic characteristics of several plant pathogenic Fusarium isolated on different hosts. Appl Entomol Phytopathol J 84:227-238

Santos CC, Domingues JL, Santos RF, Sposito M, Santos A, Novaes QS (2019) First Report of Neopestalotiopsis clavispora causing leaf spot on Macadamia in Brazil. Plant Dis: $1-4$

Publisher's note Springer Nature remains neutral with regard to jurisdictional claims in published maps and institutional affiliations. 